\title{
Solvent Effects on the UV/ Visible Absorption Spectra of Some Aminoazobenzene Dyes
}

\author{
M. S. ZAKERHAMIDI ${ }^{* 1}$, A. GHANADZADEH ${ }^{2}$ and M. MOGHADAM ${ }^{2}$ \\ ${ }^{1}$ Research Institute for Applied Physics and Astronomy, University of Tabriz, Tabriz, Iran \\ ${ }^{2}$ Department of Chemistry, Faculty of Science, University of Guilan, Rasht, Iran \\ zakerhamidi@tabrizu.ac.ir
}

Received 7 May 2012 / Accepted 18 May 2012

\begin{abstract}
Absorption spectra of three aminoazobenzene dyes with various molecular size and alkyl tails have been recorded in solvents in the range between 200 to $600 \mathrm{~nm}$. The photophysical behavior of a dissolved dye depends on the nature of its environment, i.e. the intensity, shape, and maximum absorption wavelength of the absorption band of dye in solution depends strongly on the solvent-solute interactions and solvent nature. The solvatochromic behaviors of aminoazobenzene dyes and solventsolute interactions can be analyzed by means of linear solvation energy relationships concept proposed by Kamlet and Taft. Bathochromic shift of absorption maxima of these dyes, $\lambda_{\max }$, occurs in solvent with the highest proton acceptor ability and dipolarity/polarizability.
\end{abstract}

Keywords: Aminoazobenzene dyes, Solvent-solute interactions, Linear solvation energy relationships, Solvent polarity scale

\section{Introduction}

Azo dyes are one of the important classes of colorants. The success of azo colorants is due to the simplicity of their synthesis, high molar extinction coefficient and to the medium to high light and wet fastness properties ${ }^{1-2}$. Beyond aminoazobenzene dyes importance as colorants, they are dichroic compounds with a large permanent dipole moment, long molecular structure and high order parameters in liquid crystals, giving them the potential to be applied in non-linear optical devices and LCDs ${ }^{3}$. They have high stability (both electrochemically and photo-chemically), also the advantage of high dichroic ratios because of their rod-like molecular structures ${ }^{4}$. Many studies have been studied existing Relationship between the structure and the absorption spectra and photo stability of azo dyes ${ }^{4-9}$.

Solvent effects on organic reactivity and on absorption spectra have been studied for more than a century. It is well known that the photo-physical behavior of a dissolved dye depends on the nature of its environment, i.e. the intensity, shape, and maximum absorption wavelength of the absorption band of dye in solution depends strongly on the solvent-solute interactions and solvent nature ${ }^{10-13}$. This effect is closely related to the nature and degree of dye-solvent interactions. The solvent dependent spectral shifts can 
Chem Sci Trans., 2012, 1(1), 1-8

arise from either non-specific (dielectric enrichment) or specific (e.g. hydrogen-bonding) solute-solvent interactions. The solvent effect can be determined by Solvent polarity scale or solvatochromic parameters ${ }^{14}$.

Solvent polarity is a commonly used term related to the capacity of a solvent for solvating dissolved charged or neutral, apolar or dipolar, species. Attempts to express it quantitatively have mainly involved physical solvent properties such as relative permittivity, dipole moment, or refractive index, but these parameters cannot effectively account for the multitude and specific interactions of solute-solvent on the molecularmicroscopic level ${ }^{10}$.

Spectroscopic Solvent polarity parameters have been derived from solvent sensitive standard compounds absorbing radiation in spectral ranges corresponding to UV/Vis, IR, ESR and NMR spectra ${ }^{15-19}$. In spite of the observation that single empirical parameters may serve as good approximations of solvent polarity in the sense defined, but there are many examples of solvent-sensitive processes known, which cannot be interrelated to one empirical solvent parameter.

However, multiparameter solvent polarity scale for quantitative assessment of the solvent/solute interaction and the absorption shifts can be used. The effect of solvent polarity on the absorption spectra are interpreted by means of linear solvation energy relationship (LSER) using a Kamlet-Taft Eq. $(1)^{20}$.

$$
v=v_{0}+s \pi^{*}+b \beta+a \alpha
$$

Where $\pi^{*}$ is a measure of the solvent dipolarity/polarizability ${ }^{21}, \beta$ is the scale of the solvent hydrogen bond acceptor (HBA) basicities ${ }^{22}, \alpha$ is the scale of the solvent hydrogen bond donor (HBD) acidities ${ }^{23}$ and $v_{0}$ is the regression value of the solute property in the reference solvent cyclohexane. The regression coefficients s, b and a in Eq. (1) measure the relative susceptibilities of the solvent-dependent solute property (absorption frequencies) to the indicated solvent parameters.

In this work, three aminoazobenzene, with various molecular size and alkyl tails, were studied in different solvent for understanding effects of solvents and substituents on the absorption spectra of aminoazobenzene dyes. Quantitative assessment of solvatochromic behaviors of these aminoazobenzene dyes were investigated using linear solvation energy relationship.

\section{Experimental}

Aminoazobenzene dyes (Table 1) were synthesized and purified according to the common procedure in our laboratory and used as solutes (guests) ${ }^{24}$. The solvents were purchased from Merck and the Spectroscopic Solvent polarity parameters are given in Table 2.

\section{Absorption spectroscopy}

Double beam Shimadzu UV-2450 Scan UV-Visible spectrophotometer was used to record the absorption spectra over a wavelength range 200-800 $\mathrm{nm}$ which combined with a cell temperature controller. Quartz cuvettes were used for measurements in solution via $\mathrm{l}=1 \mathrm{~cm}$. 
Table 1. Solute structure and Molecular Wight

Molecular
Weight, g/mol

Table 2. Solvent polarity parameters

\begin{tabular}{cccc}
\hline Solvent & $\pi^{*}$ & $\beta$ & $\alpha$ \\
\hline Cyclohexane & 0 & 0 & 0 \\
Butanol & 0.47 & 0.84 & 0.84 \\
1.4-Dioxan & 0.49 & 0.37 & 0 \\
Ethanol & 0.54 & 0.75 & 0.86 \\
Dicholoromethane & 0.73 & 0.1 & 0.13 \\
Acetone & 0.62 & 0.48 & 0.08 \\
DMSO & 1 & 0.76 & 0 \\
\hline
\end{tabular}

\section{Results and Discussion}

\section{The solvent effect on the absorption spectra}

The strong solvatochromic behavior can be observed for dye molecules with large dipole moment changes during transitions between two electronic states. The solvatochromic behavior of a dye is the shift of absorption wavelength due to the presence of solvent with different polarity, which is due to the interaction between the solute and solvent molecules. The visible absorption spectra of the aminoazobenzene dyes $\left(1 \times 10^{-5} \mathrm{M}\right)$ were obtained at room temperature in various organic solvents with different polarity (Figure 1). The selected spectral data are also summarized in Tables 3. The absorption spectra of the compounds in cyclohexane, which is used as reference solvent, are also shown in the aminoazobenzene dyes spectrum (Figure 1). As it can be seen, the absorption spectra of the dyes in DMSO solution are red shifted as compared to the dye spectra in other solutions, indicating relatively strong guest-host interaction between the dye molecules and the DMSO environment. From idealized theories, the solvent dielectric constant (i.e. the relative permittivity $\varepsilon_{\mathrm{r}}$ ) is often predicted to serve as a quantitative measure of solvent polarity. However, this approach is often inadequate since these theories regard solvents as a nonstructured isotropic continuum, not composed of individual solvent molecules with their own solvent-solvent interactions and they do not take into account specific solute-solvent interactions such as hydrogen-bonding and EPD/EPA interactions, which often play a dominant role in solute-solvent interactions. 
Tables 3. UV/Vis spectral data of aminoazobenzene dyes in various solvents

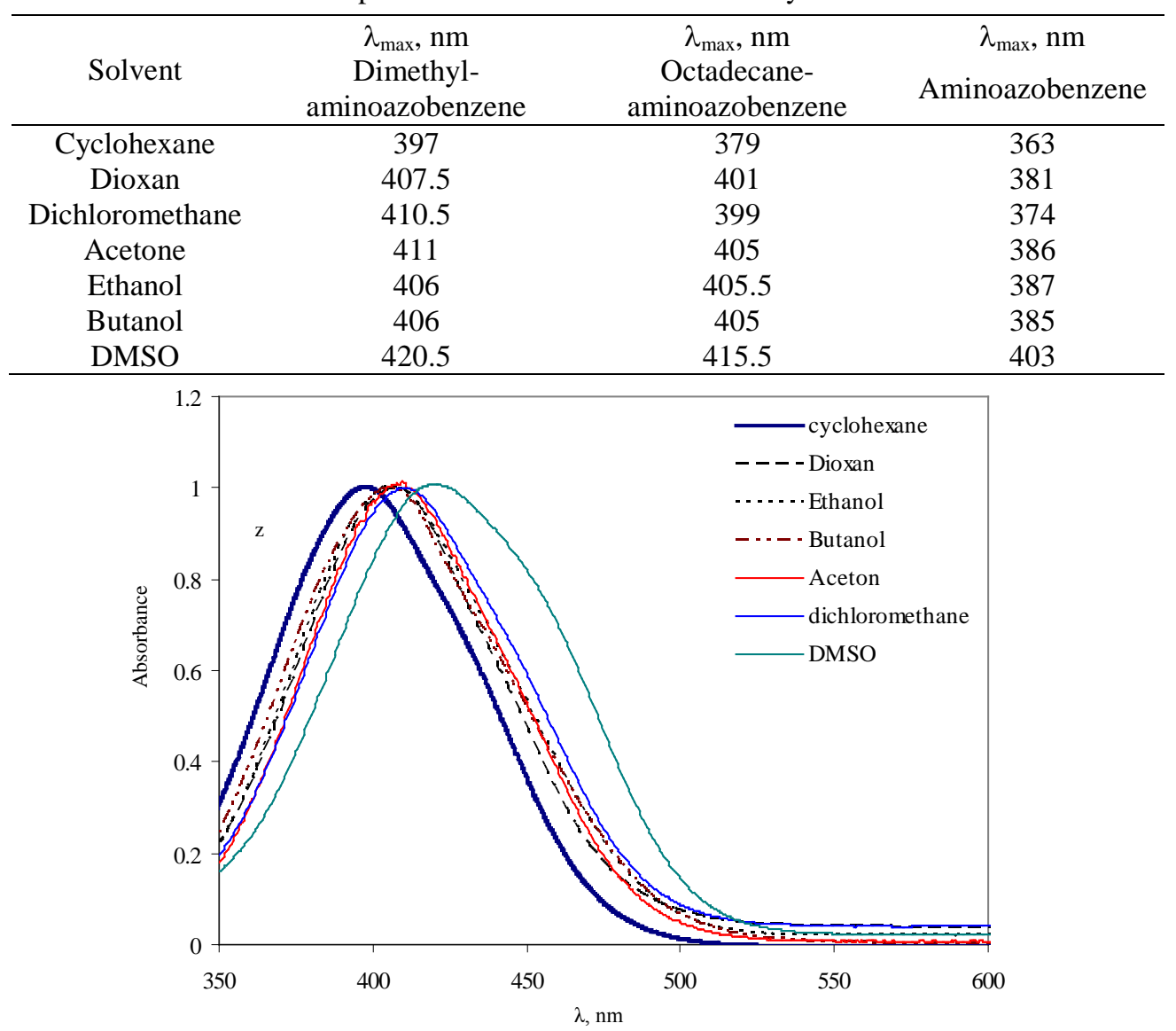

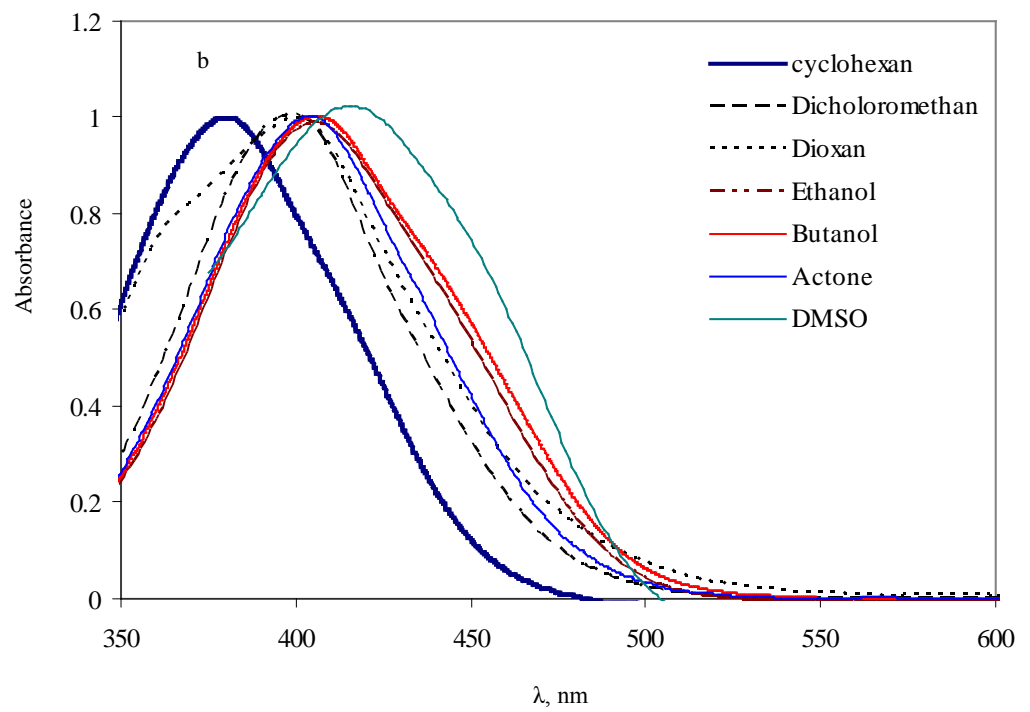




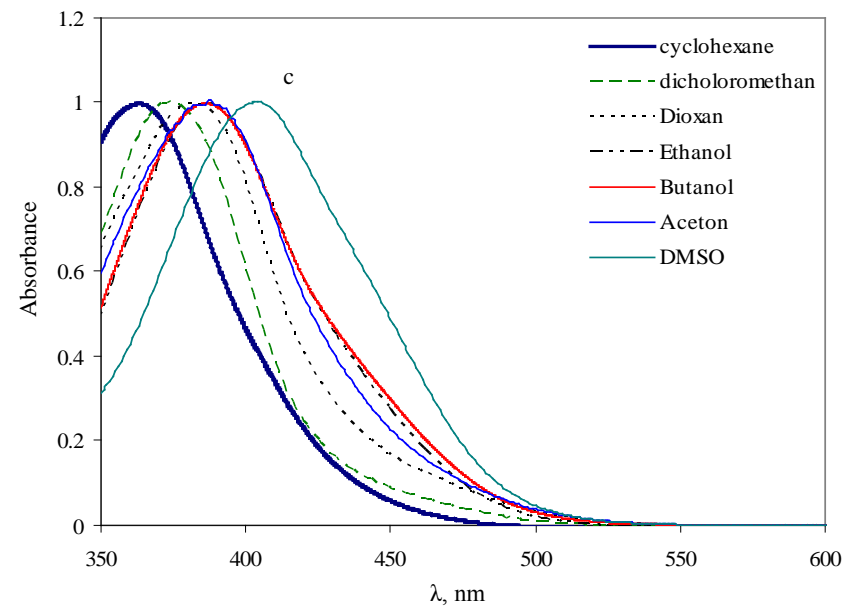

Figure 1. Visible absorption spectra of the dyes in different solvents; (a) Dimethylaminoazobenzene, (b) Octadecane-aminoazobenzene (c) Aminoazobenzene

It is obvious that such a definition of solvent polarity cannot be expressed by an individual physical quantity such as the relative permittivity (Figure 2). It means that multiparameter solvent polarity scale that correlate different solvation factors in one global equation can be used for investigation of magnitude of solute-solvent interactions on molecular and microscopic levels.
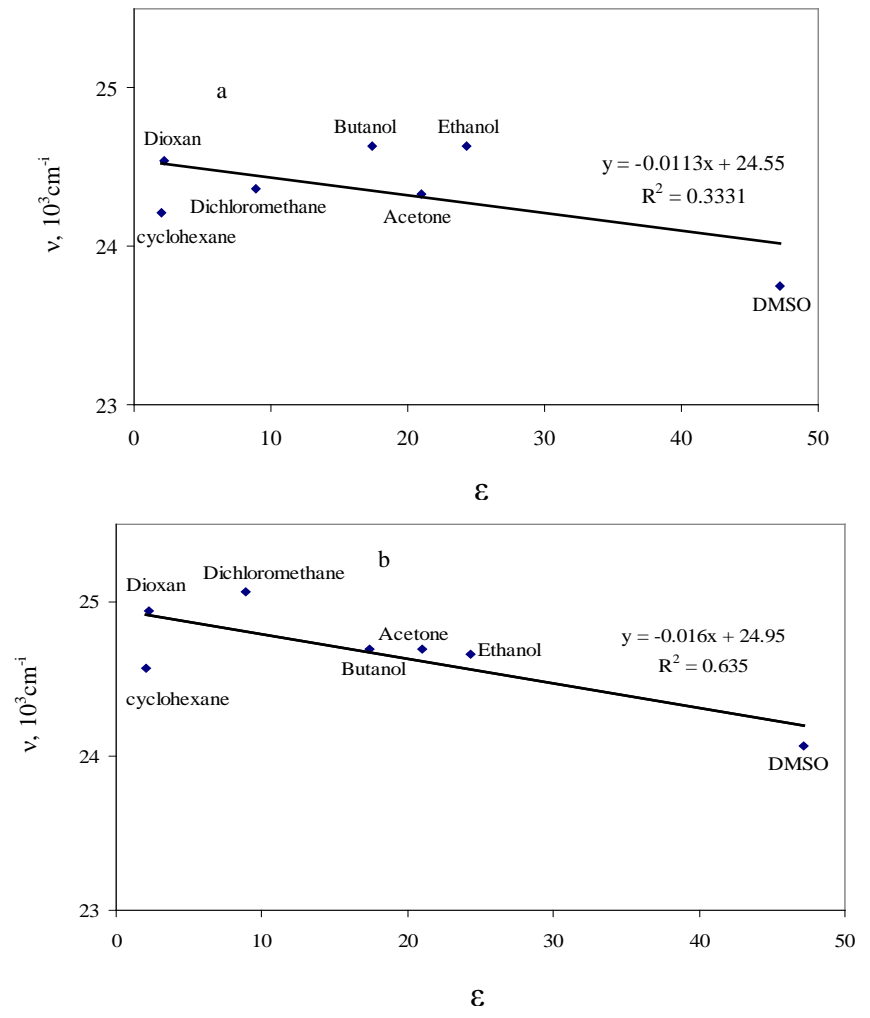


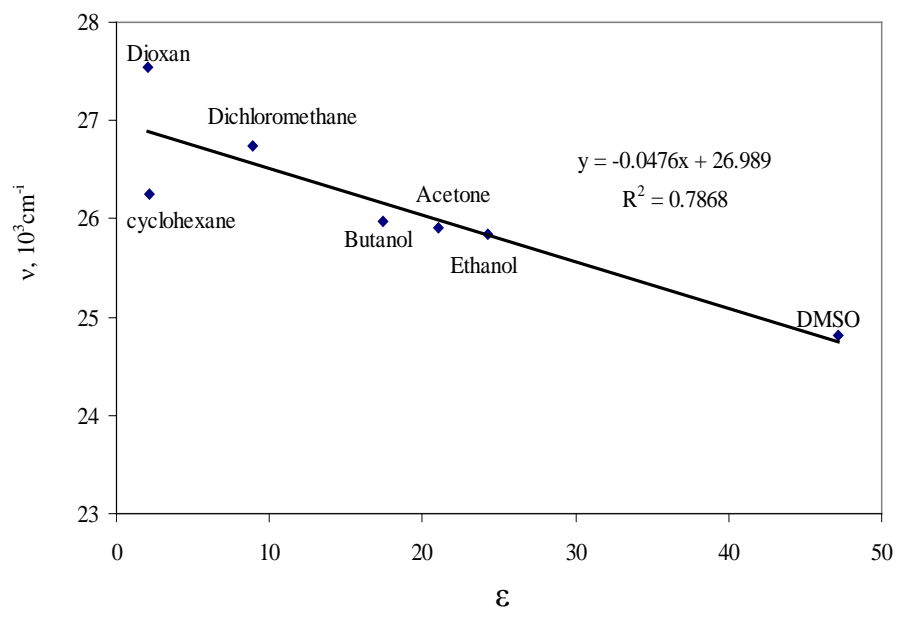

Figure 2. Variation of $v_{\max }$ of the aminoazo dyes as a function of solvent dielectric constant, (a) Dimethyl-aminoazobenzene, (b) Octadecane-aminoazobenzene and (c) Aminoazobenzene

\section{Multiparameter of solvent polarity and solvatochromic behavior}

The effects of solvent polarity and hydrogen bonding on the aminoazobenzene dyes are interpreted by means of the linear solvation energy relationship concept using Eq. (1). The solvent parameters ${ }^{10,25,26}$ are given in Table 2 . The correlations of the spectroscopic data were carried out by means of multiple linear regression analysis. It was found that absorption frequencies for azo dyes in solvents, show acceptable correlation with $\pi^{*}, \beta$ and $\alpha$ parameters. The results of the multiple regressions are presented in Tables 4 , and coefficients $v_{0}, \mathrm{~s}, \mathrm{~b}$ and a have confidence intervals at a level of significance of $95 \%$. The degree of success of Eq. (1) is shown in Figure 3 by means of a plot of $v_{\exp }$ calculated versus $v_{\text {calc }}$ observed in different solvents. The negative sign of $b$ and $s$ coefficient for all aminoazobenzene dyes indicate a bathochromic shifts with both increasing solvent hydrogen bond acceptor basicities and solvent dipolarity/polarizability. This suggests stabilization of the electronic excited state relative to the ground state. The positive sign of a coefficient for all dyes indicate a hypsochromic shifts with increasing solvent hydrogen bond donor acidities. This suggests stabilization of the ground state relative to the electronic excited state.

Table 4. Regression fits to solvatochromic parameters (Eq. (1))

\begin{tabular}{ccccccc}
\hline Dye & $v_{0}\left(10^{3} \mathrm{~cm}^{-1}\right)$ & $s\left(10^{3} \mathrm{~cm}^{-1}\right)$ & $b\left(10^{3} \mathrm{~cm}^{-1}\right)$ & $a\left(10^{3} \mathrm{~cm}^{-1}\right)$ & $R^{a}$ & $F^{b}$ \\
\hline $\begin{array}{c}\text { Dimethyl- } \\
\text { aminoazobenzene }\end{array}$ & 25.198 & -1.152 & -0.341 & 0.336 & 0.99 & 447 \\
Octadecane- & 26.259 & -1.514 & -1.084 & 0.045 & 0.97 & 36 \\
aminoazobenzene & $( \pm 0.147)$ & $( \pm 0.306)$ & $( \pm 0.358)$ & $( \pm 0.263)$ & & \\
Aminoazobenzene & 27.571 & -1.025 & -2.25 & 0.759 & 0.99 & 100 \\
& $( \pm 0.106)$ & $( \pm 0.219)$ & $( \pm 0.256)$ & $( \pm 0.188)$ & & \\
\hline \multicolumn{7}{c}{$R$-Square $(C O D),{ }^{b}$ F Statistic }
\end{tabular}




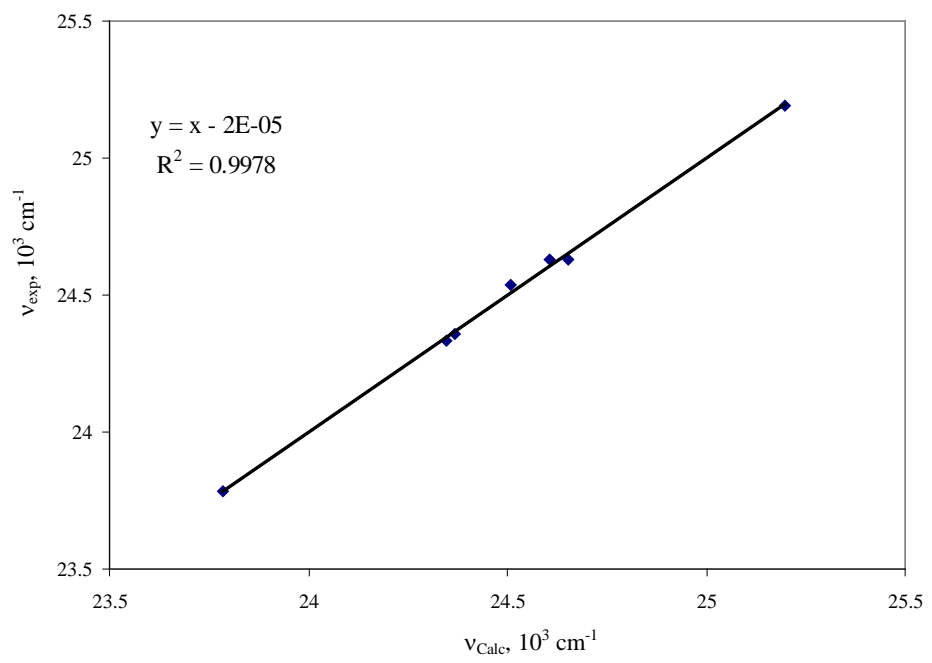

Figure 3. The plot of $v_{\max }$ observed against $v_{\max }$ calculated from Eq. (1) for dimethylaminoazobenzene in different solvents

The percentage contributions of solvatochromic parameters (Table 5) for azo dyes with electron-donating substituents in the amino group show that most of the solvatochromism is due to solvent basicities and dipolarity/polarizability rather than on the solvent acidity.

Table 5. Percentage contribution of solvatochromic parameters

\begin{tabular}{cccc}
\hline Dye & $P \pi^{*}(\%)$ & $P_{\beta}(\%)$ & $P_{\alpha}(\%)$ \\
\hline Dimethyl-aminoazobenzene & 63 & 18.6 & 18.4 \\
Octadecane-aminoazobenzene & 52 & 37 & 11 \\
Aminoazobenzene & 25.4 & 55.8 & 18.8 \\
\hline
\end{tabular}

\section{Conclusion}

These results show that the solvent effect on UV/vis absorption spectra of investigated amino azo dyes is very complex and strongly depend on the nature of the substituent on the amino group. This phenomenon is caused by the difference in the conjugational or migrating ability of the electron lone pairs on nitrogen atoms and azo-hydrazo tautomerism of amino azo dyes. These results are in accordance with structure of these dyes, (Table 1). This also indicates that the electronic behavior of the nitrogen atoms of azo group is somewhat different between derivatives with electron-donating substituents.

\section{References}

1. Zollinger H, Colour Chemistry, VCH, Weinheim, 1987.

2. Gordana S, Ušc'umlic', Dusan`Z Z . Mijin, Natas`a V. Valentic', Vlatka V. Vajs, Biljana M and Sus`I, Chem Phys Lett., 2004, 397 , 148.

3. Viswanathan N K, Kim D Y, Bian S, Williams J, Liu W, Li L, Samuelson L and Kumar J, J Mater Chem., 1999, 9 ,1941.

4. Ghanadzadeh A, Shahzamanian M A, Shoarinejad S, Zakerhamidi M S and Moghadam M, J Mole Liq., 2007, 136, 22.

5. $\quad$ Ghanadzadeh A, Zakerhamidi M S and Tajalli H, J Mole Liq., 2004, 109, 143.

6. $\quad$ Wang P Y and Wang I Y, Text Res J., 1990, 60, 519. 
7. Peng Q, Li M, Gao K and Heng L, Dyes Pigments, 1991, 15, 236.

8. $\quad$ Ertan N and Gurkan P, Dyes Pigments, 1997, 33, 137.

9. Song H, Clen K and Tian H, Dyes Pigments, 2002, 53, 257.

10. Reichardt C, Solvents and Solvent Effects in Organic Chemistry, Second edition, VCH , Weinheim, 1988.

11. DeBolt S E and Kollman P A, J Am Chem Soc., 1990, 112, 7515.

12. Fox T and Rosch N, Chem Phys Lett., 1992, 191, 33.

13. Dubois J E, Goetz E and Bienvenue A, Spectrochim Acta Part A,. 1964, 24, 1815.

14. Kessler M A and Wolfbeis O S, Spectrochim Acta Part A, 1991, 47, 187.

15. Reichardt C, Angew Chem., 1979, 91,119.

16. Kosower E M, An Introduction to Physical Organic Chemistry, Wiley, New York, 1968, 293.

17. Kamlet M J, Abboud J M, Abraham M H and Taft R W, J Org Chem., 1983, 48, 2877.

18. Hirsch J A, Concepts in Theoretical Organic Chemistry, Allyn and Bacon, Boston, 1974, 207.

19. Gutmann V, The Donor-Acceptor Approach to Molecular Interactions, Plenum Publ. Corp., New York, 1978.

20. Kamlet M J, Abboud J M and Taft R W, Prog Phys Org Chem., 1981, 13, 485.

21. Abboud J M, Kamlet M J, Taft R W, J Am Chem Soc., 1977, 99, 8325.

22. Kamlet M J, Taft R W, J Am Chem Soc., 1976, 98, 377.

23. Kamlet M J and Taft R W, J Chem Soc Perkin Trans., 1979, 2, 349.

24. Fierz-David H E D and Blangey L, The Fundamental Processes of Dye Chemistry, Fifth Edition, Interscience Publishers Inc. New York, 1949.

25. Kamlet M J, Abboud J M, Abraham M H and Taft R W, J Org Chem., 1983,48, 2877.

26. Laurence C, Nicolet P, Dalati M T, Abboud J-L M and Notario R, J Phys Chem., 1994, 98, 5807. 